\title{
The Reconstruction of Spring-Mass System with Partial Given Data
}

\author{
Wenting Wan \\ School of Mathematics and Physics, Jingchu University of Technology, Jingmen, Hubei, 448000, \\ China \\ wentingwan@163.com
}

Keywords: Spring-mass system; jacobi matrix; inverse eigenvalue problem; eigenpair

Abstract: In this paper, two inverse vibration problems of constructing a grounding spring-mass system from its two eigenpairs and part of spring stiffness are considered. The vibration system is constrained to satisfy a relation that the total mass of system is a constant, and the problems are transferred into inverse eigenvalue problems for Jacobi matrix. The necessary and sufficient conditions for the construction of physically realizable systems with positive parameters are derived. Furthermore, the corresponding numerical algorithms and numerical example are given.

\section{Introduction}

Spring-mass systems are the basic dynamic systems. Many inverse vibration problems can be divided into inverse vibration problems of spring-mass systems by using lumped mass method or finite difference method. The vibration includes the longitudinal vibration of rod, the lateral vibration of string, the torsional vibration of hub disk and so on. Inverse vibration problems for spring-mass systems, generally speaking, are how to determine the physical elements of the systems from part natural frequencies (eigenvalues) or vibration modes (eigenvectors) or some physical parameters. Related research has important application in vibration control, structural design, parameters identification, etc. The problems are transferred into inverse eigenvalue problems for Jacobi matrices in mathematics. Recently, some new results have been obtained on the inverse eigenvalue problems for Jacobi matrices, see [1-4]. Using two sets of eigenvalues or two incomplete eigenpairs, the inverse vibration problems of spring-mass systems have been studied by Nylen and Uhlig [5], and Huang, et al. [6]. Bai [7], and Tian and Dai[8] considered by one eigenpair or two eigenpairs to determine spring-mass systems, and proposed numerical algorithms for solving the problems. In view of practical engineering problems, this paper studies two classes of inverse vibration problems that are generalization of the problem in [8], and constructs the grounding spring-mass system from its two eigenpairs, some physical parameters and the total mass of system. The necessary and sufficient conditions of unique solution for the two problems are proved, moreover, the expressions of the solution and the related numerical algorithms are derived.

Assume that anterior $p$ particles of a spring-mass system are connected to the ground by springs

(Fig. 1). Generalized eigenvalue equation for the system is: $K X=\lambda M X$, where $\lambda=\omega^{2}, \omega$ is natural frequency, $X$ is vibration mode, $\lambda$ and $X$ are respectively eigenvalue and eigenvector of matrix pair $(K, M)$, particle quality $m_{i}>0$, ungrounded spring stiffness $k_{i}>0(i=1,2, \cdots, n-1)$, grounded spring stiffness $c_{j}>0(j=1,2, \cdots, p)$, mass matrix is: $M=\operatorname{diag}\left(m_{1}, m_{2}, \cdots, m_{n}\right)$, stiffness matrix is:

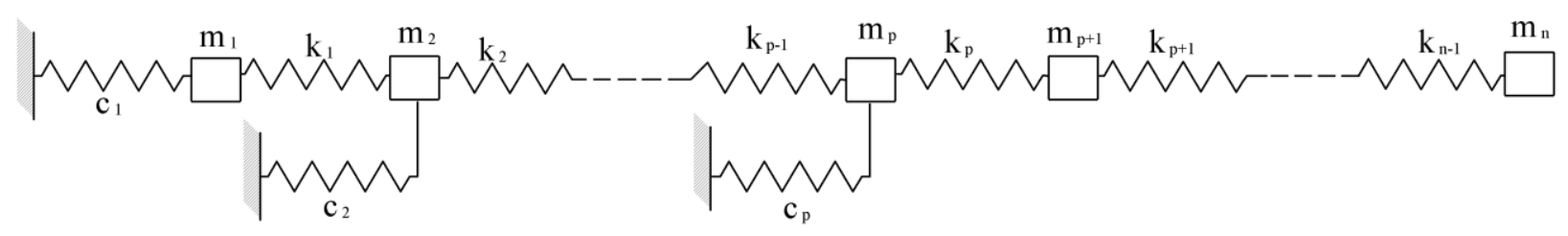

Fig. (1). A grounding spring-mass system 


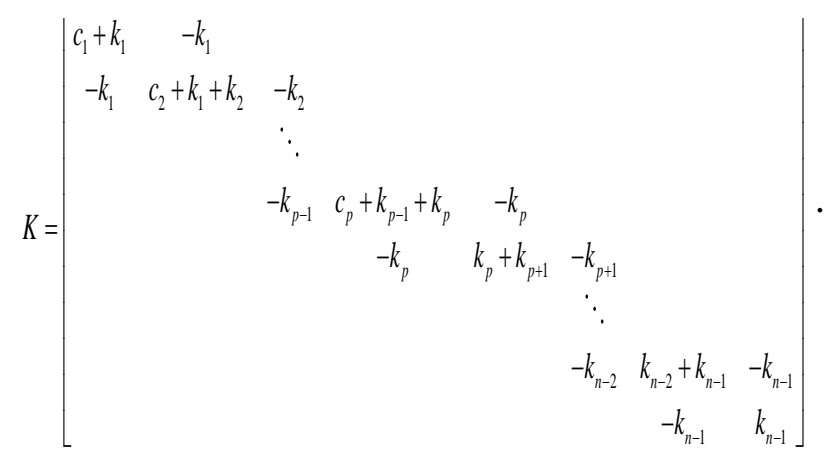

In fact, the generalized eigenvalue equation $K X=\lambda M X$ is equivalent to the standard eigenvalue equation $J X=\lambda X$, where $J=M^{-1} K$ is $n \times n$ Jacobi matrix.

The problems we considered are as follows.

Problem 1 Given two eigenpairs of the system (Fig. 1) $(\lambda, X),(\mu, Y)\left(\lambda, \mu \in \boldsymbol{R}^{+}\right.$and $\left.\lambda \neq \mu\right)$, grounded spring stiffness $\left\{c_{i}\right\}_{i=1}^{p-1}$ and constant $C$, find the remaining physical parameters of the system, that is, solve particle quality $\left\{m_{i}\right\}_{i=1}^{n}$, ungrounded spring stiffness $\left\{k_{i}\right\}_{i=1}^{n-1}$ and the $\mathrm{p}^{\text {th }}$ grounded spring stiffness $c_{p}$.

Problem 2 Given two eigenpairs of the system (Fig. 1) $(\lambda, X),(\mu, Y)\left(\lambda, \mu \in \boldsymbol{R}^{+}\right.$and $\left.\lambda \neq \mu\right)$, ungrounded spring stiffness $\left\{k_{i}\right\}_{i=1}^{p-1}$ and constant $C$, find the remaining physical parameters of the system, that is, solve particle quality $\left\{m_{i}\right\}_{i=1}^{n}$, grounded spring stiffness $\left\{c_{i}\right\}_{i=1}^{p}$ and ungrounded spring stiffness $\left\{k_{i}\right\}_{i=p}^{n-1}$.

In Problem1 and Problem 2, all parameters of the systems should satisfy

$K X=\lambda M X, K Y=\mu M Y,(1)$

and $\sum_{i=1}^{n} m_{i}=C$, where $K$ and $M$ are respectively stiffness matrix and mass matrix, $(\lambda, X)$ and $(\mu, Y)$ are respectively the $\mathrm{i}^{\text {'th }}$ and the $\mathrm{j}^{\mathrm{j}^{\text {th }}}$ eigenpair of the system $\left(1 \leq i^{\prime}, j^{\prime} \leq n\right)$, when eigenvalues of the system are arranged in ascending order, and $X=\left(x_{1}, x_{2}, \cdots, x_{n}\right)^{T}, Y=\left(y_{1}, y_{2}, \cdots, y_{n}\right)^{T} \in \boldsymbol{R}^{n}$.

Let $\quad u_{i}=x_{i}-x_{i-1}, \quad v_{i}=y_{i}-y_{i-1}, l_{i}=\lambda x_{i} v_{i}-\mu y_{i} u_{i}, i=1,2, \cdots, n$,

$h_{i}=\lambda x_{i} v_{i+1}-\mu y_{i} u_{i+1}, q_{i}=u_{i} v_{i+1}-u_{i+1} v_{i}, i=1,2, \cdots, n-1$,

$z_{i}=(\lambda-\mu) x_{i} y_{i}, f_{i}=x_{i} v_{i+1}-y_{i} u_{i+1}, g_{i}=x_{i} v_{i}-y_{i} u_{i}, w_{i}=\frac{l_{i}}{h_{i-1}} w_{i-1}+c_{i} z_{i}, t_{i}=\frac{q_{i}}{h_{i-1}} w_{i-1}+c_{i} f_{i}$,

$r_{i}=k_{i} f_{i}-k_{i-1} g_{i}, \quad s_{i}=k_{i} h_{i}-k_{i-1} l_{i}, i=1,2, \cdots, p$,

$d=\frac{f_{p}}{z_{p}}+\frac{q_{p+1}}{h_{p+1}}+\sum_{i=p+2}^{n-1} \frac{q_{i}}{h_{i}}\left[\prod_{j=p+1}^{i-1} \frac{l_{j}}{h_{j}}\right]+\frac{u_{n}}{\lambda x_{n}} \prod_{j=p+1}^{n-1} \frac{l_{j}}{h_{j}}, e=C-\sum_{i=1}^{p-1} m_{i}+\frac{k_{p-1} g_{p}}{z_{p}}$,

where $x_{0}=y_{0}=0, k_{0}=0, w_{0}=0$.

\section{Solvability of the Problems}

In this section, we will discuss the conditions for the solvability of Problem 1 and Problem 2. We first present the following definition and lemma for later discussion.

Definition. (See [9].) For $n \times 1$ real vector $X=\left(x_{1}, x_{2}, \cdots, x_{n}\right)^{T}$, sign change number of sequence $x_{1}, x_{2}, \cdots, x_{n}$ (that value being equal to zero can be neglected ) is denoted by $S(X)$.

Lemma. (See [9].) Let $\left\{\lambda_{i}\right\}_{i=1}^{n}$ be eigenvalues of $n \times n$ Jacobi matrix $J$, with $\lambda_{1}<\lambda_{2}<\cdots<\lambda_{n}$, and let $(\lambda, X)$ be eigenpair of Jacobi matrix $J$. Then, $(\lambda, X)$ is the $i^{\text {th }}$ eigenpair of $J$ if and only if $S(X)=i-1, i=1,2, \cdots, n$. 
Equations (1) can be rewritten as follows.

$\left\{\begin{array}{l}\lambda m_{i} x_{i}=c_{i} x_{i}+k_{i-1} u_{i}-k_{i} u_{i+1}, \\ \mu m_{i} y_{i}=c_{i} y_{i}+k_{i-1} v_{i}-k_{i} v_{i+1},\end{array} \quad i=1,2, \cdots, n\right.$,

where $k_{0}=k_{n}=0, x_{0}=y_{0}=0, c_{p+1}=\cdots=c_{n}=0$.

Theorem 1. Problem 1 has a unique solution if and only if

(1) $S(X)=i^{\prime}-1, S(Y)=j^{\prime}-1$;

(2) $h_{i} \neq 0$, and $h_{i}, w_{i}, t_{i}$ have the same sign, $i=1,2, \cdots, p-1 ; h_{i} \neq 0$, and $h_{i}, l_{i}, q_{i}$ have the same sign, $i=p+1, p+2, \cdots, n-1 ; x_{n} u_{n}>0, y_{n} v_{n}>0, l_{n}=0$;

(3) $d \neq 0, d$ and $e$ have the same sign;

(4) $z_{p} \neq 0$, and $z_{p}, r_{p}, s_{p}$ have the same sign.

Proof. For systems of linear equations (2), since $k_{0}=0$, if $i=1,2, \cdots, p-1$ then $\left\{\begin{array}{l}\lambda x_{i} m_{i}+u_{i+1} k_{i}=c_{i} x_{i}+u_{i} k_{i-1}, \\ \mu y_{i} m_{i}+v_{i+1} k_{i}=c_{i} y_{i}+v_{i} k_{i-1},\end{array}\right.$ have a unique solution if and only if $\left|\begin{array}{ll}\lambda x_{i} & u_{i+1} \\ \mu y_{i} & v_{i+1}\end{array}\right| \neq 0$, that is $h_{i} \neq 0, i=1,2, \cdots, p-1$, and the solution is $k_{i}=\frac{l_{i} k_{i-1}+c_{i} z_{i}}{h_{i}}, m_{i}=\frac{q_{i} k_{i-1}+c_{i} f_{i}}{h_{i}}$, Let $w_{i}^{\prime}=l_{i} k_{i-1}+c_{i} z_{i}$, then $k_{i}=\frac{w_{i}^{\prime}}{h_{i}}$, thus, $k_{i-1}=\frac{w_{i-1}^{\prime}}{h_{i-1}}$,

Therefore, $w_{i}^{\prime}=\frac{l_{i}}{h_{i-1}} w_{i-1}^{\prime}+c_{i} z_{i}$, Obviously, $w_{i}^{\prime}$ and $w_{i}$ have the same form.

Let $w_{i}=w_{i}^{\prime}$, then $k_{i}=\frac{w_{i}}{h_{i}}$. And $q_{i} k_{i-1}+c_{i} f_{i}=\frac{q_{i}}{h_{i-1}} w_{i-1}+c_{i} f_{i}=t_{i}$, thus, $k_{i}=\frac{w_{i}}{h_{i}}, m_{i}=\frac{t_{i}}{h_{i}}, i=1,2, \cdots, p-1$.

And for $k_{i}>0, m_{i}>0, h_{i}, w_{i}, t_{i}$ have the same sign, $i=1,2, \cdots, p-1$.

For systems of linear equations (2), if $i=p$ and assume that $k_{p}$ is given, then $\left\{\begin{array}{l}\lambda x_{p} m_{p}-x_{p} c_{p}=k_{p-1} u_{p}-k_{p} u_{p+1}, \\ \mu y_{p} m_{p}-y_{p} c_{p}=k_{p-1} v_{p}-k_{p} v_{p+1},\end{array}\right.$ have a unique solution if and only if $\left|\begin{array}{ll}\lambda x_{p} & -x_{p} \\ \mu y_{p} & -y_{p}\end{array}\right| \neq 0$, that is $z_{p} \neq 0$, and the solution is $m_{p}=\frac{r_{p}}{z_{p}}, c_{p}=\frac{s_{p}}{z_{p}}$.

And for $m_{p}>0, c_{p}>0, z_{p}, r_{p}, s_{p}$ have the same sign.

In addition, the expressions of $m_{p}$ and $c_{p}$ can be expanded as follows.

$$
m_{p}=k_{p} \frac{f_{p}}{z_{p}}-\frac{k_{p-1} g_{p}}{z_{p}}, c_{p}=k_{p} \frac{h_{p}}{z_{p}}-\frac{k_{p-1} l_{p}}{z_{p}} \text {. }
$$

For systems of linear equations (2), if $i=p+1, p+2, \cdots, n-1$ and assume that $k_{p}$ is given, then $\left\{\begin{array}{l}\lambda x_{i} m_{i}+u_{i+1} k_{i}=k_{i-1} u_{i}, \\ \mu y_{i} m_{i}+v_{i+1} k_{i}=k_{i-1} v_{i},\end{array}\right.$ have a unique solution if and only if $\left|\begin{array}{cc}\lambda x_{i} & u_{i+1} \\ \mu y_{i} & v_{i+1}\end{array}\right| \neq 0$, that is $h_{i} \neq 0$, and the solution is

$$
k_{i}=k_{i-1} \frac{l_{i}}{h_{i}}, m_{i}=k_{i-1} \frac{q_{i}}{h_{i}}, \quad i=p+1, p+2, \cdots, n-1 .
$$

And for $k_{i}>0, m_{i}>0, h_{i}, l_{i}, q_{i}$ have the same sign, $i=p+1, p+2, \cdots, n-1$. 
In addition, the expressions of $k_{i}$ and $m_{i}$ can be expanded as follows.

$$
\begin{aligned}
& k_{i}=k_{i-1} \frac{l_{i}}{h_{i}}=k_{i-2} \frac{l_{i-1}}{h_{i-1}} \cdot \frac{l_{i}}{h_{i}}=\cdots=k_{p} \prod_{j=p+1}^{i} \frac{l_{i}}{h_{j}}, i=p+1, p+2, \cdots, n-1, \\
& m_{p+1}=k_{p} \frac{q_{p+1}}{h_{p+1}}, \quad m_{i}=k_{i-1} \frac{q_{i}}{h_{i}}=k_{i-2} \frac{l_{i-1}}{h_{i-1}} \cdot \frac{q_{i}}{h_{i}}=\cdots=k_{p} \frac{q_{i}}{h_{i}} \prod_{j=p+1}^{i-1} \frac{l_{i}}{h_{j}} .
\end{aligned}
$$

For systems of linear equations (2), if $i=n$ and assume that $k_{p}$ is given, then $\left\{\begin{array}{l}\lambda x_{n} m_{n}=k_{n-1} u_{n}, \\ \mu y_{n} m_{n}=k_{n-1} v_{p},\end{array}\right.$ have a unique solution if and only if $m_{n}=\frac{k_{n-1} u_{n}}{\lambda x_{n}}=\frac{k_{n-1} v_{n}}{\mu y_{n}}$.

Since $m_{n}>0, k_{n-1}>0, \lambda>0, \mu>0, x_{n} u_{n}>0, y_{n} v_{n}>0$, and $\lambda x_{n} v_{n}=\mu y_{n} u_{n}$, that is $l_{n}=0$.

Therefore, $m_{n}=\frac{k_{n-1} u_{n}}{\lambda x_{n}}$ or $\frac{k_{n-1} v_{n}}{\mu y_{n}}$

In addition, using the recurrence formula of $k_{n-1}$, the expression of $m_{n}$ can be expanded as follows. $m_{n}=k_{n-1} \frac{u_{n}}{\lambda x_{n}}=k_{p} \frac{u_{n}}{\lambda x_{n}} \prod_{j=p+1}^{n-1} \frac{l_{i}}{h_{j}} . \quad$ From $\sum_{i=1}^{n} m_{i}=C$ and the above expansions of $m_{i}(i=p, p+1, \cdots, n)$, we have $\sum_{i=1}^{p-1} m_{i}+k_{p} \frac{f_{p}}{z_{p}}-\frac{k_{p-1} g_{p}}{z_{p}}+k_{p} \frac{q_{p+1}}{h_{p+1}}+k_{p} \sum_{i=p+2}^{n-1} \frac{q_{i}}{h_{i}}\left[\prod_{j=p+1}^{i-1} \frac{l_{j}}{h_{j}}\right]+k_{p} \frac{u_{n}}{\lambda x_{n}} \prod_{j=p+1}^{n-1} \frac{l_{j}}{h_{j}}=C$, that is $k_{p}\left\{\frac{f_{p}}{z_{p}}+\frac{q_{p+1}}{h_{p+1}}+\sum_{i=p+2}^{n-1} \frac{q_{i}}{h_{i}}\left[\prod_{j=p+1}^{i-1} \frac{l_{j}}{h_{j}}\right]+\frac{u_{n}}{\lambda x_{n}} \prod_{j=p+1}^{n-1} \frac{l_{j}}{h_{j}}\right\}=C-\sum_{i=1}^{p-1} m_{i}+\frac{k_{p-1} g_{p}}{z_{p}}$

Therefore, $k_{p}=\frac{e}{d}$.

Since $k_{p}>0, d \neq 0, d$ and $e$ have the same sign. Combining with above discussion and with lemma, we get the theorem.

It is not difficult to prove Theorem 2 by the same method which we apply to prove Theorem 1.

Theorem 2. Problem 2 has a unique solution if and only if

(1) $S(X)=i^{\prime}-1, S(Y)=j^{\prime}-1$;

(2) $z_{i} \neq 0$, and $z_{i}, r_{i}, s_{i}$ have the same sign, $i=1,2, \cdots, p-1 ; h_{i} \neq 0$, and $h_{i}, l_{i}, q_{i}$ have the same sign, $i=p+1, p+2, \cdots, n-1 ; x_{n} u_{n}>0, y_{n} v_{n}>0, l_{n}=0$;

(3) $d \neq 0, d$ and $e$ have the same sign;

(4) $z_{p} \neq 0$, and $z_{p}, r_{p}, s_{p}$ have the same sign.

When the above-mentioned conditions are satisfied, the remaining physical parameters of spring-mass system have the expressions as follows:

$$
\begin{aligned}
& m_{i}=\frac{r_{i}}{z_{i}}, \quad c_{i}=\frac{s_{i}}{z_{i}}, i=1,2, \cdots, p-1, \quad m_{p}=\frac{r_{p}}{z_{p}}, c_{p}=\frac{s_{p}}{z_{p}}, k_{p}=\frac{e}{d}, k_{i}=k_{i-1} \frac{l_{i}}{h_{i}}, \\
& m_{i}=k_{i-1} \frac{q_{i}}{h_{i}}, i=p+1, p+2, \cdots, n-1, m_{n}=\frac{k_{n-1} u_{n}}{\lambda x_{n}} \text { or } \frac{k_{n-1} v_{n}}{\mu y_{n}} .
\end{aligned}
$$

\section{Numerical Method}

Based on the above discussion, we write numerical algorithm for solving Problem 1 as follows.

\section{Algorithm 1.}

Step 1. Compute $S(X), S(Y)$. If $S(X) \neq i^{\prime}-1$ or $S(Y) \neq j^{\prime}-1$, go to step 6 .

Step 2. Compute $\left\{u_{i}\right\}_{i=1}^{n},\left\{v_{i}\right\}_{i=1}^{n},\left\{l_{i}\right\}_{i=1}^{n},\left\{h_{i}\right\}_{i=1}^{n-1},\left\{q_{i}\right\}_{i=1}^{n-1},\left\{f_{i}\right\}_{i=1}^{p},\left\{z_{i}\right\}_{i=1}^{p},\left\{w_{i}\right\}_{i=1}^{p-1},\left\{t_{i}\right\}_{i=1}^{p-1}$. 
Step 3. If some $h_{i}=0, i=1,2, \cdots, p-1, p+1, \cdots, n-1$, go to step 6;

If $h_{i}, w_{i}, t_{i}(i=1,2, \cdots, p-1)$ have different sign, go to step 6 ;

If $h_{i}, l_{i}, q_{i}(i=p+1, p+2, \cdots, n-1)$ have different sign, go to step 6 ;

If $x_{n} u_{n} \leq 0$ or $y_{n} v_{n} \leq 0$ or $l_{n} \neq 0$, go to step 6 .

Step 4. Compute $\left\{k_{i}\right\}_{i=1}^{p-1},\left\{m_{i}\right\}_{i=1}^{p-1}, d, e$. If $d=0$ or $d, e$ have different sign, go to step 6 .

Step 5. Compute $k_{p}, r_{p}, s_{p}$. If $z_{p}=0$ or $z_{p}, r_{p}, s_{p}$ have different sign, go to step 6.

Step 6. The solution can not be determined uniquely, end the algorithm.

Step 7. Compute $m_{p}, c_{p},\left\{k_{i}\right\}_{i=p+1}^{n-1},\left\{m_{i}\right\}_{i=p+1}^{n-1}, m_{n}$.

Example 1. Given $\lambda=1.0508, \mu=2.1186, n=8, p=3,\left\{c_{i}\right\}_{i=1}^{2}=\{3,2\}$,

$X=(-0.6041,-0.5581,-0.4094,-0.3459,-0.0691,0.1601,0.0170,-0.0754)^{T}$,

$Y=(-0.1911,0.1296,0.4811,0.2395,-0.3766,0.3127,-0.5350,0.3635)^{T}$, and $\sum_{i=1}^{8} m_{i}=C=35$,

construct $K$ and $M$ such that $(\lambda, X)$ and $(\mu, Y)$ are respectively the $3^{\text {rd }}$ and the $6^{\text {th }}$ eigenpair of the system.

By Algorithm 1, we get $S(X)=2, S(Y)=5$.

$\left\{u_{i}\right\}_{i=1}^{8}=\{-0.6041,0.0460,0.1487,0.0635,0.2768,0.2292,-0.1431,-0.0924\}$,

$\left\{v_{i}\right\}_{i=1}^{8}=\{-0.1911,0.3207,0.3515,-0.2416,-0.6161,0.6893,-0.8477,0.8985\}$,

$\left\{l_{i}\right\}_{i=1}^{8}=\{-0.1233,-0.2007,-0.3028,0.0556,0.2656,-0.0359,-0.1773,0\}$,

$\left\{h_{i}\right\}_{i=1}^{7}=\{-0.1850,-0.2470,0.0392,0.0835,0.1328,-0.0478,-0.0887\}$,

$\left\{q_{i}\right\}_{i=1}^{7}=\{-0.1849,-0.0315,-0.0582,0.0278,0.3320,-0.0957,-0.2069\}$,

$\left\{f_{i}\right\}_{i=1}^{3}=\{-0.1849,-0.2154,0.0684\}, \quad\left\{z_{i}\right\}_{i=1}^{3}=\{-0.1233,0.0772,0.2103\}$,

$\left\{w_{i}\right\}_{i=1}^{2}=\{-0.3698,-0.2468\},\left\{t_{i}\right\}_{i=1}^{2}=\{-0.5548,-0.4939\}$.

By (3), we have $\left\{k_{i}\right\}_{i=1}^{2}=\{1.9995,0.9995\},\left\{m_{i}\right\}_{i=1}^{2}=\{2.9999,1.9999\}$,

hence $d=9.6480, e=28.9764$. By (7), we obtain $k_{3}=3.0033$, hence $r_{3}=0.4206, s_{3}=0.4204$.

By (4), (5), (6), we have $m_{3}=2.0001, c_{3}=1.9989,\left\{k_{i}\right\}_{i=4}^{7}=\{2,3.9991,3.0012,6.0016\}$,

$\left\{m_{i}\right\}_{i=4}^{7}=\{0.9984,4.9993,8.0012,7.0021\}, m_{8}=6.9992$.

Using Matlab, it is easy to get that all generalized eigenvalues of $K X=\lambda M X$ are $\sigma(K, M)=\{0.0247,0.3799,1.0508,1.3319, \quad 1.9117,2.1188,3.0928,6.4857\}$.

The eigenvector which corresponds to eigenvalue $\lambda=1.0508$ is $X=(-0.6041,-0.5581,-0.4094,-0.3459,-0.0691,0.1601,0.0170,-0.0754)^{T}$, and the eigenvector which corresponds to eigenvalue $\mu=2.1188$ is $Y=(-0.1909,0.1295,0.4807,0.2392,-0.3764,0.3127,-0.5354,0.3639)^{T}$.

Obviously, $\sum_{i=1}^{8} m_{i}=35.0001$. The numerical value explain that the algorithm 1 is efficient.

The algorithm is presented for solving Problem 2 as follows.

\section{Algorithm 2.}

Step 1. Compute $S(X), S(Y)$. If $S(X) \neq i-1$ or $S(Y) \neq j-1$, go to step 6 .

Step 2. Compute $\left\{u_{i}\right\}_{i=1}^{n},\left\{v_{i}\right\}_{i=1}^{n},\left\{l_{i}\right\}_{i=1}^{n},\left\{h_{i}\right\}_{i=1}^{n-1},\left\{z_{i}\right\}_{i=1}^{p},\left\{f_{i}\right\}_{i=1}^{p},\left\{g_{i}\right\}_{i=1}^{p},\left\{r_{i}\right\}_{i=1}^{p-1},\left\{s_{i}\right\}_{i=1}^{p-1},\left\{q_{i}\right\}_{i=p+1}^{n-1}$.

Step 3. If some $z_{i}=0$ or $z_{i}, r_{i}, s_{i}(i=1,2, \cdots, p-1)$ have different sign, go to step 6;

If some $h_{i}=0$ or $h_{i}, l_{i}, q_{i}(i=p+1, p+2, \cdots, n-1)$ have different sign, go to step 6;

If $x_{n} u_{n} \leq 0$ or $y_{n} v_{n} \leq 0$ or $l_{n} \neq 0$, go to step 6 . 
Step 4. Compute $\left\{m_{i}\right\}_{i=1}^{p-1},\left\{c_{i}\right\}_{i=1}^{p-1} d, e$. If $d=0$ or $d, e$ have different sign, go to step 6. Step 5. Compute $k_{p}, r_{p}, s_{p}$. If $z_{p}=0$ or $z_{p}, r_{p}, s_{p}$ have different sign, go to step 6 .

Step 6. The solution can not be determined uniquely, end the algorithm.

Step 7. Compute $m_{p}, c_{p},\left\{k_{i}\right\}_{i=p+1}^{n-1},\left\{m_{i}\right\}_{i=p+1}^{n-1}, m_{n}$.

\section{Conclusion}

This paper discusses the constructional problems for the spring-mass system whose anterior $\mathrm{p}$ particles are connected to the ground. Two eigenpairs and anterior p-1 grounded spring stiffness or anterior p-1 ungrounded spring stiffness to determine the real vibration system are solved under the total mass of system constraint. The necessary and sufficient conditions for the existence and uniqueness of the solution are obtained. The results of numerical example show that the proposed algorithm work well.

\section{Acknowledgment}

This work is supported by the Key Scientific Research Project of Hubei Provincial Department of Education (No. D20134301), the Teaching Research Project of Jingchu University of Technology (No. JX2014-19).

\section{References}

[1] X.Q. Wu. A divide and conquer algorithm on the double dimensional inverse eigenvalue problem for Jacobi matrices, Appl. Math. Comput., vol. 219, no. 8 (2012), p. 3840-3846.

[2] G.S. Guseinov. On an inverse problem for two spectra of finite Jacobi matrices, Appl. Math. Comput., vol. 218, no. 14 (2012), p. 7573-7589.

[3] Y. Wei. A Jacobi matrix inverse eigenvalue problem with mixed data, Linear Algebra Appl., vol. 439, no. 10 (2013), p. 2774-2783.

[4] Y. Wei. Inverse eigenvalue problem of Jacobi matrix with mixed data, Linear Algebra Appl., vol. 466 (2015), p. 102-116.

[5] P. Nylen, and F. Uhlig. Inverse eigenvalue problems associated with spring-mass systems, Linear Algebra Appl., vol. 254, no. 1-3 (1997), p. 409-425.

[6] X.T. Huang, X.Y. Hu, and L. Zhang. Physical parameters reconstruction of a fixed-fixed mass-spring system from its characteristic data, J. Comput. Appl. Math., vol. 206, no. 2 (2007), p. 645-655.

[7] Z.J. Bai. Constructing the physical parameters of a damped vibrating system from eigendata, Linear Algebra Appl., vol. 428, no. 2-3 (2008), p. 625-656.

[8] X. Tian, and H. Dai. Inverse mode problems for the finite element model of a vibrating rod, Appl. Math. Comput., vol. 214, no. 2 (2009), p. 479-486.

[9] G.M.L. Gladwell. Inverse Problems in Vibration, $2^{\text {nd }}$ ed., Kluwer Academic Publishers: Dordrecht, the Netherland (2004). 